International Journal of Distributed and Parallel Systems (IJDPS) Vol.3, No.4, July 2012

\title{
Performance Evaluation of AODV and DSR ROUTING PRotocols IN MANET NETWORKS
}

\author{
Ammar Odeh, Eman AbdelFattah and Muneer Alshowkan \\ Department of Computer Science \& Engineering, University of Bridgeport \\ Bridgeport, CT 06604, USA \\ $\{$ Aodeh, eman, malshowk\}@bridgeport.edu
}

\begin{abstract}
Extensive use of wireless networks in different fields increases the need to improve their performance, as well as minimize the amplitude of loss messages. Device mobility, where there is no standard topology that can be applied or fixed routing that can be designed, is a topic that received recent attention in wireless networks.

In a Mobile Ad Hoc Network (MANET), some nodes may join the network while others may leave. In this paper, we analyze a MANET's performance for two proactive protocols; Ad Hoc On-Demand Distance Vector (AODV) Protocol, and Dynamic Source Routing (DSR) Protocol. By using network simulator $N S 2$, we setup and evaluate the performance of AODV and DSR protocols with respect to the packets' size.
\end{abstract}

\section{KEYWORDS}

Routing Protocols, Wireless Network, Mobile Network,Ad Hoc, MANET, NS2, DSR, AODV, Simulation.

\section{INTRODUCTION}

\subsection{Background}

Wireless networks provide connection flexibility between users in different places. Moreover, the network can be extended to any place or building without the need for a wired connection. Wireless networks are classified into two categories; Infrastructure networks and Ad Hoc networks as shown in Figure 1.

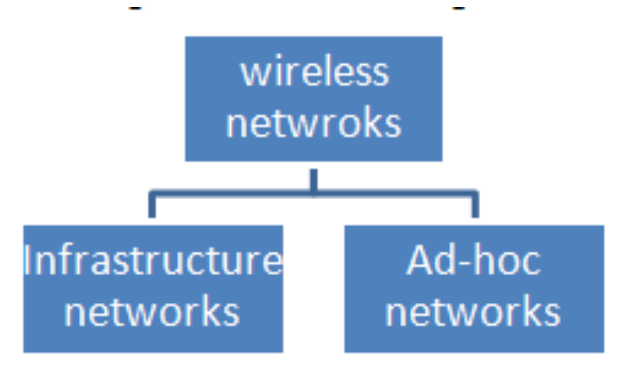

Figure1: Wireless Networks Categories

\subsubsection{Infrastructure networks}

An Access Point (AP) represents a central coordinator for all nodes. Any node can be joining the network through AP. In addition, AP organizes the connection between the Basic Set Services (BSSs) so that the route is ready when it is needed. However, one drawback of using DOI : $10.5121 /$ ijdps.2012.3402 
an infrastructure network is the large overhead of maintaining the routing tables. Figure 2 shows an infrastructure network.

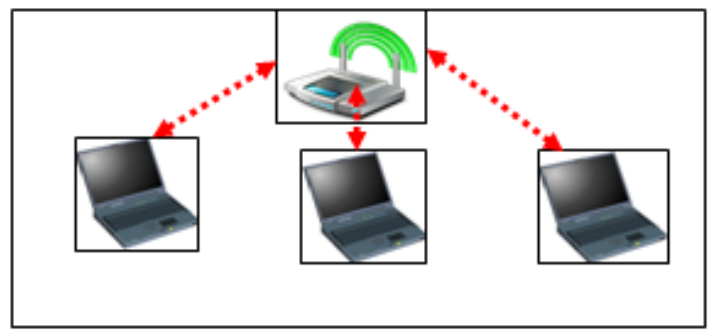

Figure 2: An Infrastructure Network

\subsubsection{Ad Hoc networks}

Ad Hoc networks do not have a certain topology or a central coordination point. Therefore, sending and receiving packets are more complicated than infrastructure networks. Figure 3 illustrates an Ad Hoc network.

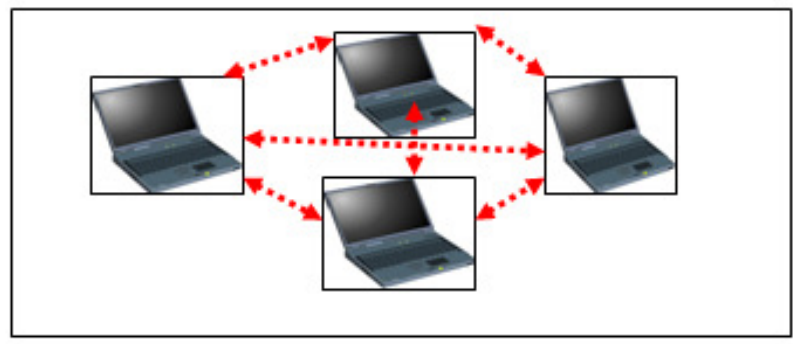

Figure 3: An Ad Hoc Network

Nowadays, with the immense growth in wireless network applications like handheld computers, PDAs and cell phones, researchers are encouraged to improve the network services and performance. One of the challenging design issues in wireless Ad Hoc networks is supporting mobility in Mobile Ad Hoc Networks (MANETs). The mobility of nodes in MANETs increases the complexity of the routing protocols and the degree of connection's flexibility. However, the flexibility of allowing nodes to join, leave, and transfer data to the network pose security challenges.

\subsection{Motivation}

A routing protocol plays a key role to measure the performance of a MANET. Routing protocols are classified under two categories;proactive protocols and reactive protocols as shown in Figure 4. When nodes move over time from one position to another, it's less efficient to use proactive(Table driven) protocols where route will be already established before a packet is sent. Therefore, reactive (On-Demand)protocolssuch as Dynamic Source Routing (DSR)protocol are more appropriate to be used in MANET networks.

In our research, we present two well-known reactive routing protocols; AODV and DSR. We evaluate the effect of packets' size on a MANET performance.

This paper is organized as follows; section II discusses some prior works for measurement of MANET performance, some performance metrics applied in our simulation are discussed in section III and in section IV, we present experimental results of AODV and DSR protocols. Finally, a conclusion is presented in section V. 


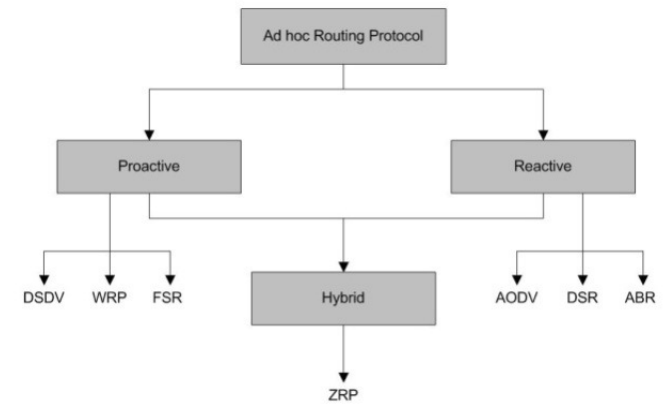

Figure 4: Routing Protocols

\section{PRIOR WORK}

Verma et. al. [1] discusses the MANET performance over AODV protocol. The authors focus on two parameters, Packet Delivery Fraction (PDF) and Average End to End Delay.

Where

$$
\begin{gathered}
P D F=(\text { Actual Received Packet/Actual send packet }) * 100 \% \\
\text { Average End to End Delay }=\text { End to End delay/ Received Packet }
\end{gathered}
$$

The authors conclude that the performance of AODV routing protocol over Transmission Control Protocol (TCP) is more efficient than Constant Bit Rate (CBR). Thus, TCP traffic is better than CBR traffic.

A comparison among AODV, DSR and ADV (proactive protocols) is presented in [2]. The authors conclude that an Adaptive distance vector (ADV) protocol provides lower connection time for TCP connection. Also, it provides higher throughput than DSR and AODV protocols with respect to the number of TCP connections over CBR traffic. On the other hand, ADV has higher overhead in bps than DSR and AODV [3].

Paul et. al. [4], include an interesting analysis of AODV and DSR performance over Vehicle Area Network (VANET) by studying the node density and node speed. The lower density and speed produce higher packet delivery ratio (PDR). Moreover, packet loss delay is reduced.The authors discuss the variation of DSR and AODV with respect to TCP and CBR. For example, when the node speed is high, the packet delivery ratio for AODV under TCP is average.

Asma et. al. [5] evaluate three routing protocols which are DSDV, AODV and DSR. The DSDV has low throughput but also has high routing load compared to AODV and DSR. Both AODV and DSR protocols perform very well. Although in some situations AODV outperforms DSR, DSR has the best performance especially when evaluated based on the average end to end delay. Moreover, changing the packet size doesn't affect the performance of DSDV but affects the performance of AODV and DSR. All protocols perform well when they are evaluated based on the mobility of the nodes.

In the Monarch Project [6], the authors provide a comprehensive study about AODV, DSDV, TORA and DSR protocols. The study considers the periodic advertisements, On-demand routediscovery, hop-by-hop routing and source routing, and the usage of the feedback from MAC layer when there is a failure. The authors conclude that DSR is performing better than the other routing protocols at different mobility factors such as the mobility rate and the movement speeds. 
International Journal of Distributed and Parallel Systems (IJDPS) Vol.3, No.4, July 2012

In [7] the authors compare two routing protocols. The first protocol is the reactive Dynamic MANET On-demand (DYMO). The second protocol is the proactive Optimized Link State Routing (OLSR). The comparison is based on the packet delivery ratio, the average end-to-end delay and the normalized routing overhead. The results show that the performance of DYMO is better than OLSRin both MANET and VANET networks.

In [8], the authors study DYMO routing protocol with respect to average end-to-end throughput, average end-to-end delay, packet delivery ratio, routing overhead and path optimality. The authors compare AODV and DYMO routing protocols. They conclude that DYMO routing protocol performs better than AODV as it is being able to handle different mobility ranges and various traffic patterns.

\section{Performance Metrics}

The following parameters are used in our simulation to compare the performance of AODV and DSR routing protocols over a MANETnetwork.

- Sending time: time consumed to send packet from MAC to application layer.

- Accessing time: waiting time at MAC layer to access the transmission channel.

- Transmission time: time required to send a packet from the transmitter to the receiver through the physical layer.

$$
\text { Packet transmission time }=\text { Packet size } / \text { Bit rate }
$$

- Propagation time: the time required for the signal to traverse through wireless link from transmitter to receiver.

$$
\text { Propagation time }=\text { distance } / \text { Speed Of Light }
$$

- Reception time: the time when a packet is received at MAC layer of the receiver side.

- Receive time: the time when a packet is received at the application layer of the receiver side.

- Aggregate throughput: is the average of successful packets delivery via a communication link.

- Drop packet: it occurs when packet loss during travelling from source to destination.

- Efficiency of routing protocol

\section{Simulation and Results}

$$
\text { Efficiency }=(\text { No. of acknowledged packets } / \text { No. of transmitted packets }) * 100
$$

This section describes the experimental tools setup and parameters used in simulation of a MANET network. Ubuntu 11 is used as the operating system because it is user friendly which makes it easy to manage. Network Simulation 2 (NS2.35) is used as simulation software which runs smoothly over Ubuntu 11. In Table 1, we describe MANET parameters that are used in this simulation to measure its performance and compare it with different protocols over a MANETnetwork.In our simulation, we are studying the relation between different MANET performance parameters with respect to packets' size.

Figure 5 shows the relation between packets' size and sending time. As the packets' size increases, the sending time increases. In the simulation, AODV protocol uses less amount of sending time compared to DSR.

In Figures 6, 7 and 8 AODV and DSR protocols achieve comparable results in accessing time, transmission time, and propagation time as the packets' size increases.

In Figure 9, we observe that throughput rapidly increases when the packets' size increases. On the other hand, in Figure 10 the amount of dropped packets increases when the packets' size increases. 
International Journal of Distributed and Parallel Systems (IJDPS) Vol.3, No.4, July 2012

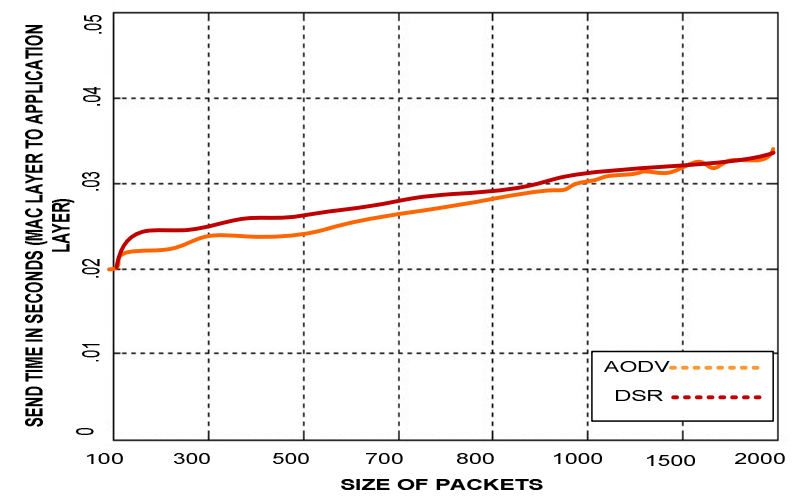

Figure 5: Relation between packets' size and sending time over AODV and DSR

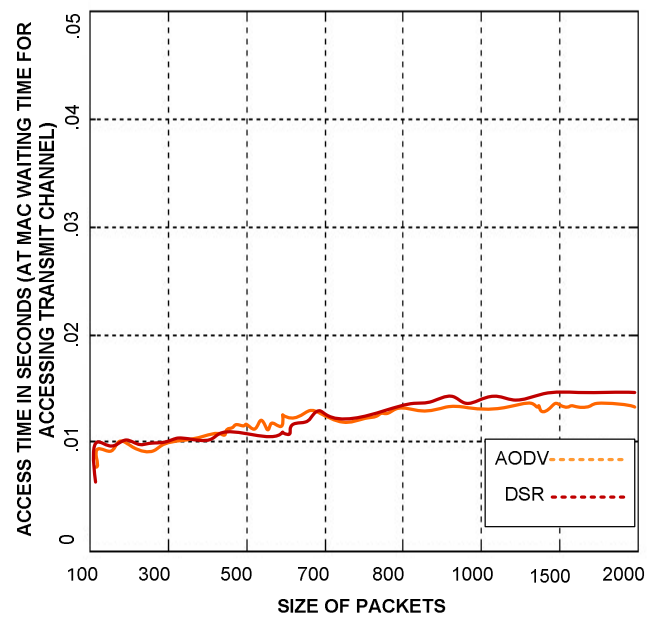

Figure 6: Relation between packets' size and accessing time over AODV and DSR

Table 1: Parameters used in simulation

\begin{tabular}{|c|c|}
\hline PARAMETER & VALUE \\
\hline Operating system & Ubuntu 11 \\
\hline NS2-2 Version & 2.35 \\
\hline Channel type & Wireless Channel \\
\hline Number of nodes & 15 \\
\hline Speed & $3,5,7,10,20,25$ \\
\hline Data type & UDP/CBR \\
\hline Simulation Time & 160 seconds \\
\hline MAC protocol & MAC/802.11 \\
\hline Data packet size & 100,300, 500, 700, 800, 1000, 1500, 2000 \\
\hline Area of simulation & TwoR 700 \\
\hline Radio Propagation Model & AODV/DSR \\
\hline Routing Protocol & \\
\hline
\end{tabular}


International Journal of Distributed and Parallel Systems (IJDPS) Vol.3, No.4, July 2012

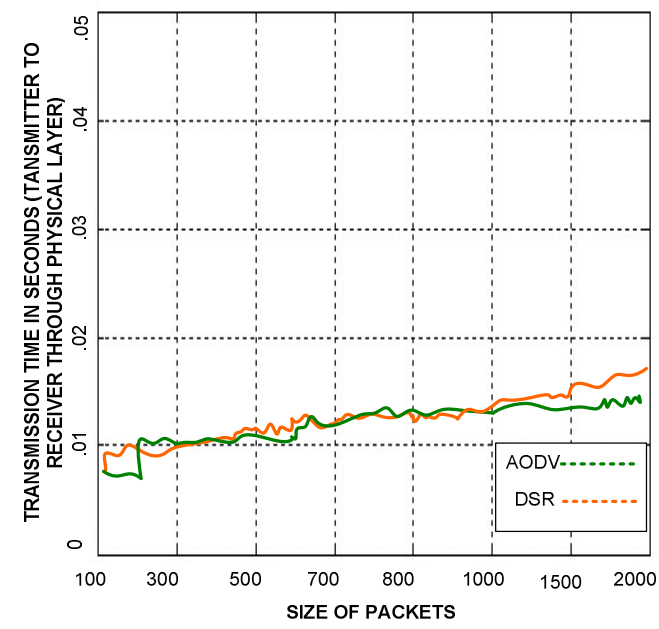

Figure 7: Relation between packets' size and transmission time over AODV and DSR

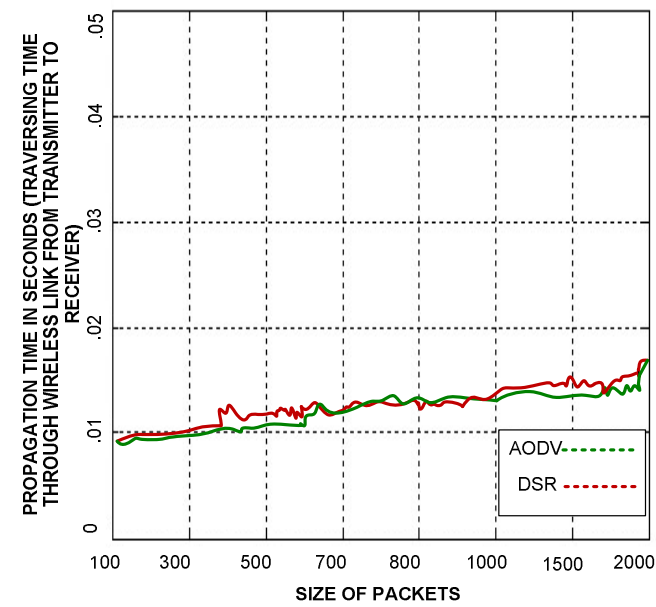

Figure 8: Relation between packets' size and propagation time over AODV and DSR

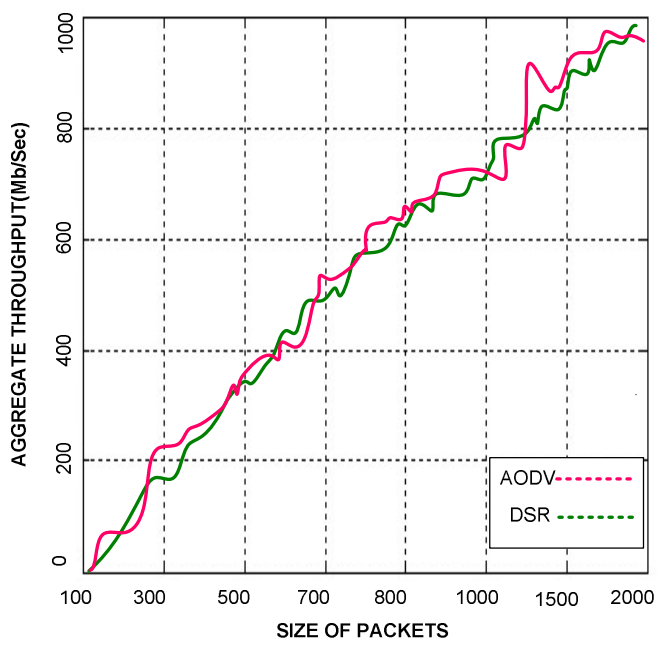

Figure 9: Relation between packets' size and throughput over AODV and DSR 


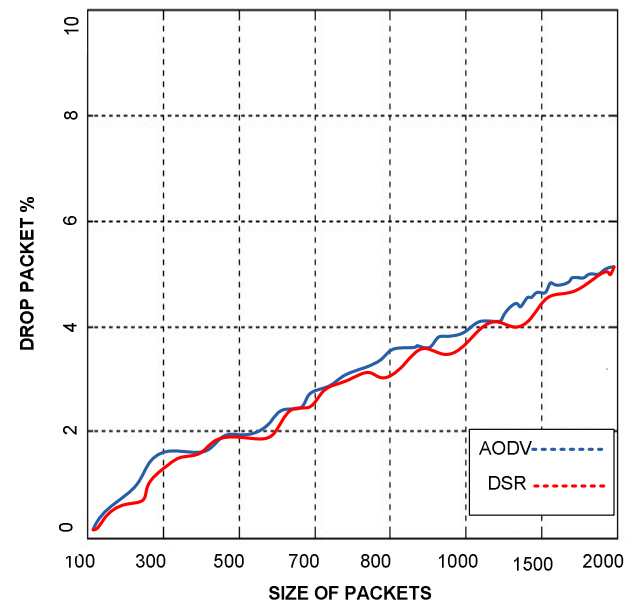

Figure 10: Relation between packets' size and dropping packets over AODV and DSR

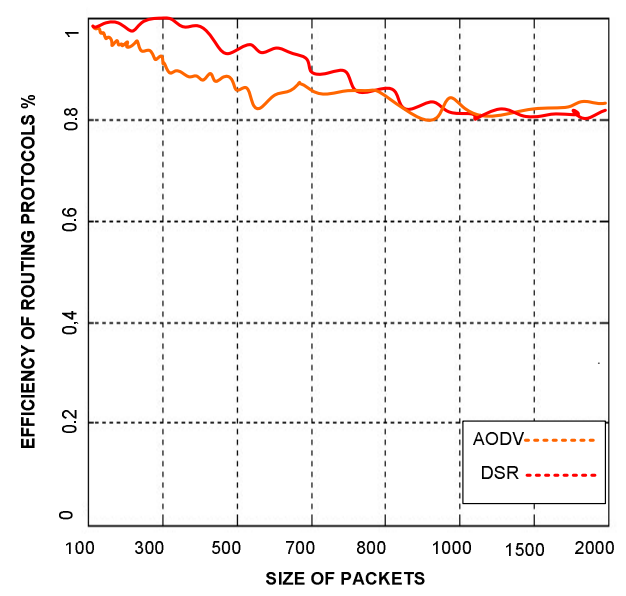

Figure11: Relation between packets' size and efficiency over AODV and DSR

Figure 11 shows the protocols' efficiency where a small packet size leads to better performance and an increase in the packets' size decreases the efficiency.

Figures 12 and 13 show the relation between packets' size, and transmitted and received traffic for DSR and AODV protocols respectively. It can be observed that the performance of both protocols is comparable. 
International Journal of Distributed and Parallel Systems (IJDPS) Vol.3, No.4, July 2012

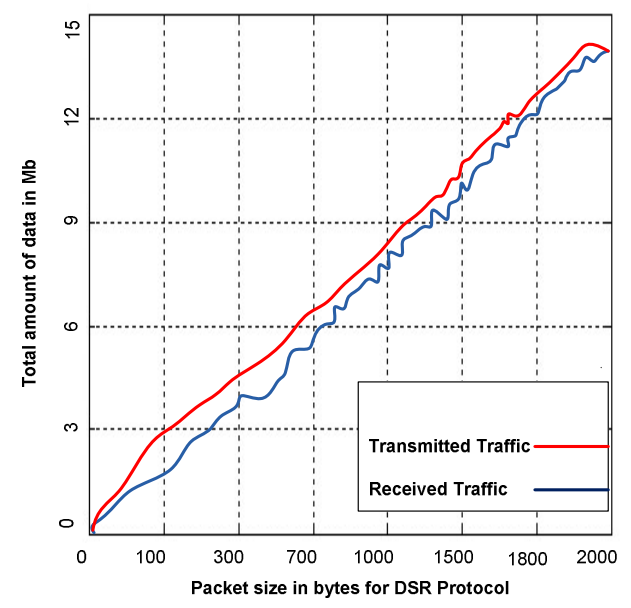

Figure12: Relation between packets' size and transmitted and received traffic for DSR protocol

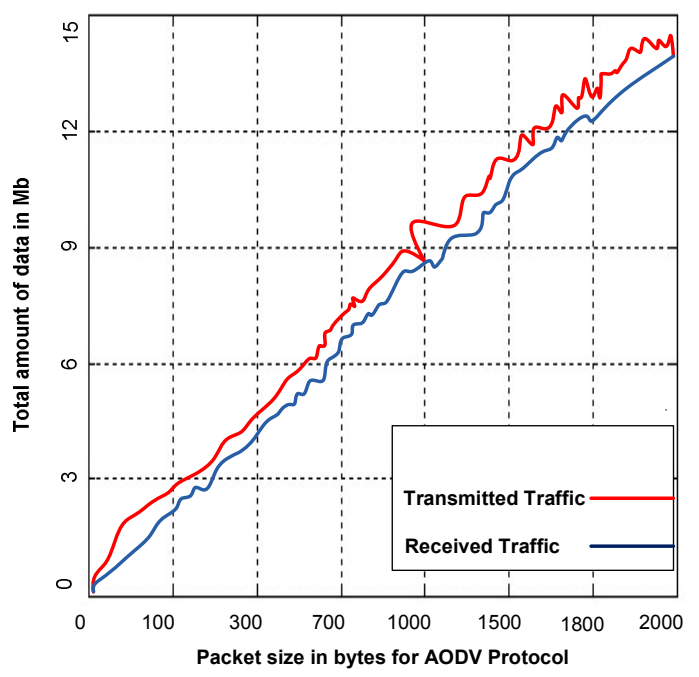

Figure13: Relation between packets' size and transmitted and received traffic for AODV protocol

\section{Conclusions}

In this paper, we studied the performance of two MANET reactive routing protocols; AODV and DSR. The different performance metrics were investigated with respect to packets' size.

In our experiments, DSR has shown better performance in terms of efficiency for a packet size less than 700 bytes. However, both protocols have illustrated comparable results for other performance metrics.

It is expected that our future studies will investigate the performance of proactive protocols versus the performance of reactive protocols and the possibility of introducing a new hybrid protocol that capitalizes on the strengths of both categories.

\section{ACKNOWLEDGEMENTS}

We would like to express our special thanks of gratitude to our friend Abdul Razaque who supports and guides us during our research. 
International Journal of Distributed and Parallel Systems (IJDPS) Vol.3, No.4, July 2012

\section{REFERENCES}

[1] D.Verma, D.Chandrawanshi, "Comparative Performance Evaluation of AODV over CBR and TCP Traffic, International Journal of Computer Science and Technology," Vol. 2, Issue 2, June 2011.

[2] T. Dyer, R. Boppana, “A Comparison of TCP Performance over Three Routing Protocols for Mobile Ad Hoc Networks, ACM Symposium on Mobile Ad Hoc Networking \& Computing (Mobihoc)," October 2001.

[3] S. Gupta, Ashish Chourey, "PERFORMANCE EVALUATION OF AODV PROTOCOLUNDER PACKET DROP ATTACKS IN MANET," International Journal of Research in Computer Science ISSN 2249-8265 Vol. 2, Issue 1, 2011.

[4] B. Paul, Md. Ibrahim, Md. Bikas, "Experimental Analysis of AODV \& DSR over TCP \& CBR Connections with Varying Speed and Node Density in VANET," International Journal of Computer Applications (0975 - 8887) Vol. 24, No.4, June 2011.

[5] T. Asma, G. Rajneesh, T. Sunil, "Comparative Performance Analysis of DSDV, AODV and DSR Routing Protocols in MANET using NS2," in International Conference on Advances in Computer Engineering, Bangalore, Karnataka, India, 2010, 330.

[6] J. Broch, D. A. Maltz, D. B. Johnson, Y. Hu, J. Jetcheva, “A Performance Comparison of MultiHop Wireless Ad Hoc Network Routing Protocols," in Fourth Annual ACM/IEEE International Conference on Mobile Computing and Networking, Ballas, Texas, 1998.

[7] S. Sagar, J. Saqib, A. Bibi, N. Javaid, "Evaluating and Comparing the Performance of DYMO and OLSR in MANETs and in VANETs," in Multitopic Conference (INMIC), 2011 IEEE 14th International, Karachi, Pakistan, 2011, 362-366.

[8] M. Quan-xing, X. Lei, "DYMO Routing Protocol Research and Simulation Based on NS2," in International Conference on Computer Application and System Modeling, Taiyuan, Shanxi, China, 2010, V14-41.

\section{Authors}

Ammar Odeh is a $\mathrm{PhD}$. Student at University of Bridgeport. He earned the M.S. degree in Computer Science College of King Abdullah II School for Information Technology (KASIT) at the University of Jordan in Dec. 2005 and the B.Sc. in Computer Science from the Hashemite University. He has worked as a Lab Supervisor in Philadelphia University (Jordan) and Lecturer in Philadelphia University for the ICDL courses and as technical

Eman Abdelfattah had received the P Degree in Computer Science from the University of Bridgeport in 2011. Also, she had received the MS Degree in Computer Science from the University of Bridgeport in 2002. She worked as a programmer and computer teacher in several places in the period from 1983 to 2000. She worked as a $\mathrm{C}++$ and Java instructor in the Continuing Education Department, Housatonic Community College, Bridgeport, Connecticut. Currently, she is working as an adjunct instructor at the University of Bridgeport, Connecticut.

She has research interests in the areas of networking and communications. Her

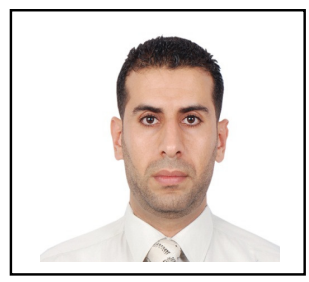
research results were published in prestigious international conferences in circuits and VLSI design. She actively participated as a committee member of the International Conferences on Engineering Education, Instructional Technology, Assessment, and E-learning EIAE 05, EIAE 06, EIAE 07, EIAE 08, EIAE 09and EIAE 10.

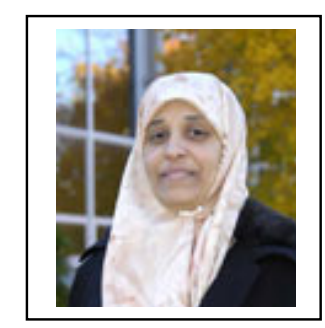


International Journal of Distributed and Parallel Systems (IJDPS) Vol.3, No.4, July 2012

Muneer Alshowkan, is currently pursuing a Ph.D. degree in Computer Science and Engineering at the University of Bridgeport. In 2011, he received his Master's degree in Information, Network and Computer Security at New York Institute of Technology, and received his B.S. in Computer and Management Systems at King Faisal University in Kingdom of Saudi Arabia. He is currently an active member of the IEEE.

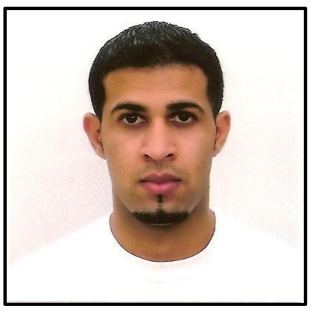

